\title{
Imaging of Vitrified Biological Specimens by Confocal Cryo-Fluorescence Microscopy and Cryo-FIB/SEM Tomography
}

\author{
Alexander Rigort ${ }^{1}$, Robert Kirmse ${ }^{1}$, Volker Döring ${ }^{1}$, Michael Schwertner ${ }^{2}$ and Ralf Wolleschensky ${ }^{1}$ \\ 1. Carl Zeiss Microscopy, Jena, Germany, Email: alexander.rigort@ zeiss.com \\ ${ }^{2 .}$ Linkam Scientific Instruments, Tadworth, UK
}

The investigation of vitrified biological specimens (i.e. samples that are plunge or high-pressure-frozen) enables the visualization of cellular ultrastructure in a near-native fully hydrated state, unadulterated by harmful preparation methods. Here, we focus on two recent cryo-imaging modalities and discuss their impact on cryo-correlative workflows. First, we present confocal cryo-fluorescence microscopy, utilizing a novel confocal detector scheme with improved signal-to-noise ratio (SNR) and resolution. Second, we show volume imaging of multicellular specimens by cryo-focused ion beam scanning electron microscopy (FIB/SEM) .

Confocal laser scanning microscopes (LSM) are renowned for their optical sectioning capability, a feature enabled by utilizing a pinhole that rejects out-of-focus light. Closing the pinhole improves lateral resolution, but also causes less light to reach the detector leading to reduced signal-to-noise ratios. In cryo-fluorescence microscopy the situation is aggravated by the fact that currently no immersion optics are available and consequently only numerical apertures below NA 1 are possible. Here we combined Airyscan, a novel detector module (available for ZEISS LSM 780, 800 and 880) together with a cryocorrelative stage (Linkam CMS196) for cryo-fluorescence imaging of vitrified Dictyostelium discoideum cells prepared on electron microscopy grids. The Airyscan detection module allows the spatiallyresolved detection of fluorescence light otherwise rejected by the pinhole in a standard confocal system. We show that under cryo-conditions even without immersion optics a significant increase in resolution and SNR can be obtained with Airyscan compared to standard confocal images (Figure 1).

In FIB/SEM tomography three-dimensional volumetric data from biological specimens is obtained by sequentially removing material with the ion beam and imaging the milled block faces by scanning with the electron beam. Only recently, it has been shown that this imaging method can be applied also to frozen-hydrated specimens [1]. Cryo-FIB/SEM tomography allows the mapping of large multicellular specimens in the near-native state and is particularly suited to analyze samples that require remaining hydrated. We demonstrate this approach by imaging the cryo-immobilized nematode Pristionchus pacificus (Figure 2).

Both methods by themselves promise significant advantages for biomedical research by being able to investigate biological specimens in the near-native fully hydrated state. Yet correlating both imaging modalities, LSM and FIB/SEM of vitrified samples, has the potential to provide even deeper insights into biological context. In contrast to correlative workflows using resin-embedded samples, cryoimaging workflows do not have to find the optimal balance between preserving cellular ultrastructure and maintaining the functional integrity of fluorophores. Moreover, major mechanisms leading to irreversible bleaching of fluorescent molecules are suppressed at cryo temperatures [2]. The correlation between cryo light and electron microscopy data will greatly benefit from an increase in resolution in fluorescence imaging. Cryo-Airyscan is a first step in that direction and can deliver three-dimensional 
optical sectioning data that can be used to reliably target cellular structures in a FIB/SEM microscope, before the structural context is explored by cryo-FIB/SEM tomography.

\section{References:}

[1] Schertel A et al., "Cryo FIB-SEM: volume imaging of cellular ultrastructure in native frozen specimens.” J Struct Biol. 2013 Nov;184(2):355-60. doi: 10.1016/j.jsb.2013.09.024.

[2] Kaufmann R, Hagen C, Grunewald K. Fluorescence cryo-microscopy: current challenges and prospects. Current opinion in chemical biology. 2014;20:86-91. DOI: 10.1016/j.cbpa.2014.05.007

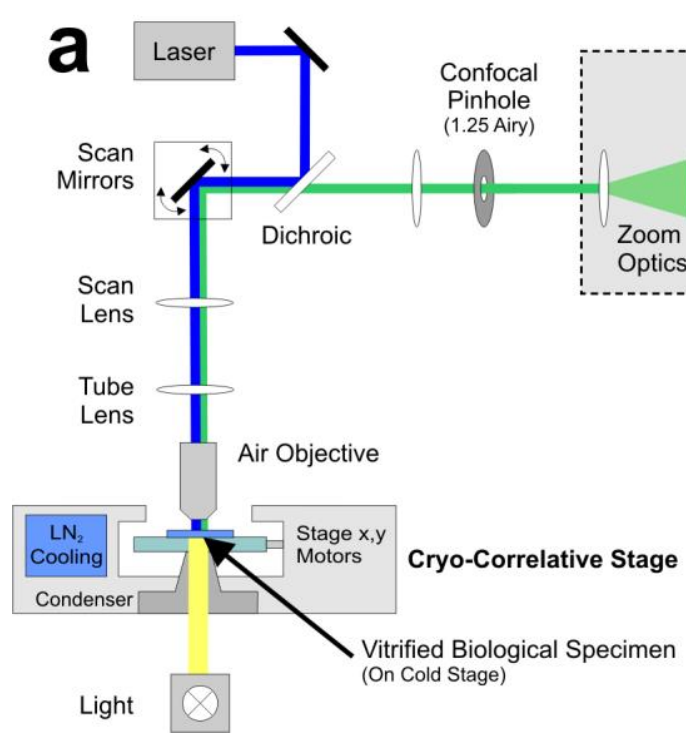

Figure 1. Confocal cryo-fluorescence beampath (a). Comparison between confocal cryo-fluorescence (b) and Airyscan (c) image of vitrified D. discoideum cells expressing mRFP-Tubulin. Scale bar: $10 \mu \mathrm{m}$.
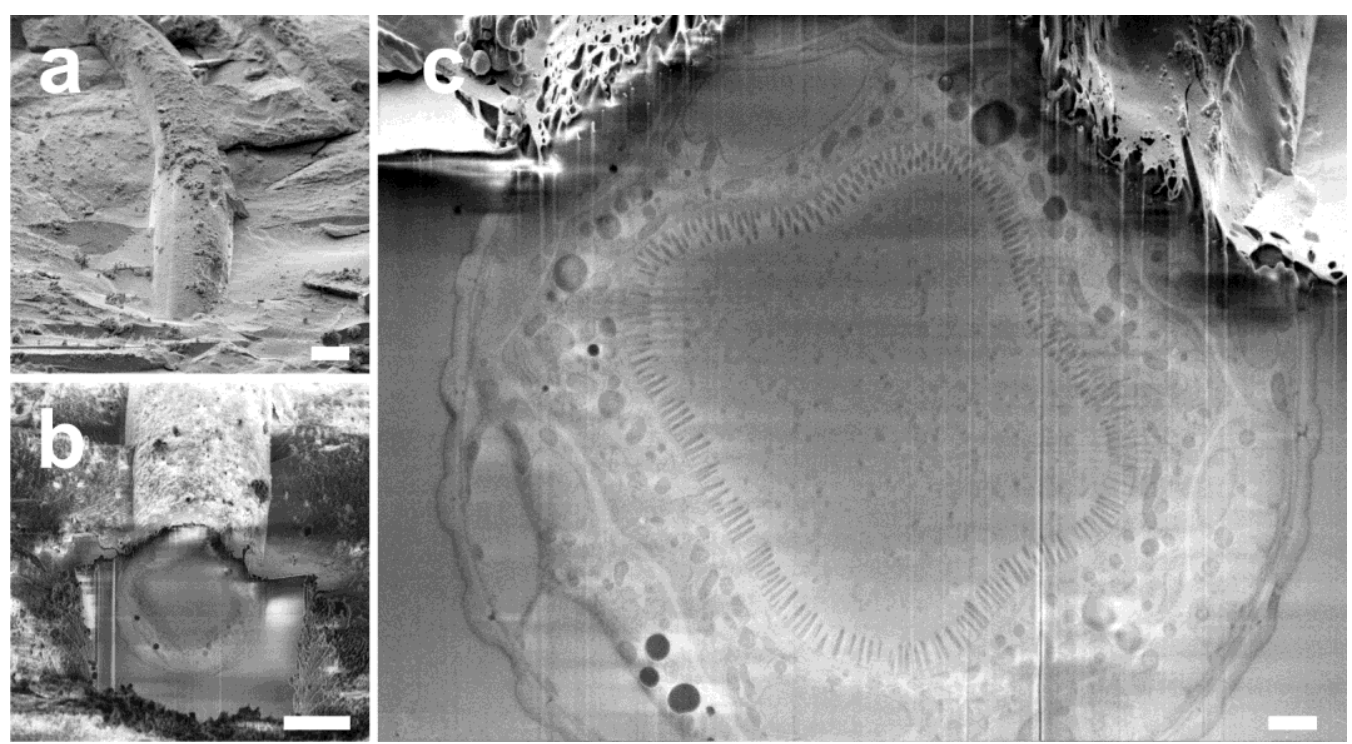

Figure 2. Cryo-FIB/SEM tomography of high-pressure frozen P. pacificus (a). In-lens SEM micrographs showing the milled cryo-block faces exhibiting a cross-sectional view of the worms' intestine (b,c). Scale bars: (a,b) $10 \mu \mathrm{m}$, (c) $1 \mu \mathrm{m}$. 